\title{
Highly connected manifolds with positive Ricci curvature
}

\author{
ChARles P BOYER \\ KRZYSZTOF GALICKI
}

\begin{abstract}
We prove the existence of Sasakian metrics with positive Ricci curvature on certain highly connected odd dimensional manifolds. In particular, we show that manifolds homeomorphic to the $2 \mathrm{k}$-fold connected sum of $S^{2 n-1} \times S^{2 n}$ admit Sasakian metrics with positive Ricci curvature for all $\mathrm{k}$. Furthermore, a formula for computing the diffeomorphism types is given and tables are presented for dimensions 7 and 11 .
\end{abstract}

53C25; 57R55

\section{Introduction}

An important problem in global Riemannian geometry is that of describing the class of manifolds that admit metrics of positive Ricci curvature. The only known obstructions for obtaining such metrics come from either the classical Myers theorem or the obstructions to the existence of positive scalar curvature which is fairly well understood (see the recent review article by Rosenberg and Stolz [36] for discussion and references). Given the lack of obstructions it seems most natural to develop techniques for proving the existence of positive Ricci curvature metrics. Over the years several methods for doing so have appeared. These include symmetry methods, bundle constructions, surgery theory, algebro-geometric techniques. We refer the reader to recent papers (Boyer, Galicki and Nakamaye [13; 15], Grove and Ziller [23] and Schwachhöfer and Tuschmann [37]) for a discussion of the history and pertinent references. In [13; 15], with M Nakamaye, we introduced a method for proving the existence of positive Ricci curvature on odd dimensional manifolds which relies on a transverse version of Yau's famous proof of the Calabi conjecture. The odd dimensional manifolds to which this method has been applied are hypersurfaces of isolated singularities coming from weighted homogeneous polynomials. All such manifolds are what are sometimes called "highly connected". So far, with collaborators, we have applied our methods successfully mainly to rational homology spheres $[4 ; 11 ; 7]$, homotopy spheres [15; 9; 10], and connected sums of $S^{2} \times S^{3}$ [14; 12; 5]; (see also Kollár [28]). A recent review of our method can be found in [6]. 
The purpose of this note is to prove the existence of Sasakian metrics of positive Ricci curvature on certain odd dimensional highly connected smooth manifolds. Manifolds of dimension $2 n$ or $2 n+1$ that are $n-1$ connected are often referred to as highly connected manifolds. They are relatively tractable and there is a classification of such manifolds by C T C Wall [41; 42] and his students (Barden [1] and Wilkens [43]) as well as Crowley [18]. Our first result concerns dimension $4 n-1$, where we prove the following:

Theorem 1 Let $n \geqslant 2$ be an integer, then for each positive integer $k$ there exist Sasakian metrics with positive Ricci curvature in $D_{n}(k)$ of the $\left|b P_{4 n}\right|$ oriented diffeomorphism classes of the $(4 n-1)$-manifolds $2 k \#\left(S^{2 n-1} \times S^{2 n}\right)$ that bound a parallelizable manifold, where the number $D_{n}(k)$ is determined by the explicit formula (A-4) given in the Appendix. In particular, $D_{n}(1)=\left|b P_{4 n}\right|$ for all $n \geqslant 2$, so $2 \#\left(S^{2 n-1} \times S^{2 n}\right)$ admits Sasakian metrics of positive Ricci curvature in every oriented diffeomorphism class.

Here $k \#\left(M_{1} \times M_{2}\right)$ denotes the $k$-fold connected sum of the manifold $M_{1} \times M_{2}$. In the case of the connected sums of products of standard spheres, metrics of positive Ricci curvature have been constructed previously by Sha and Yang [39]. However, the existence of such metrics for the exotic differential structures appears to be new. In dimension $4 n+1$ we prove a somewhat weaker result:

Theorem 2 For each pair of positive integers $(n, k)$ there exists an oriented $(2 n-1)-$ connected $(4 n+1)$-manifold $K$ with $H_{2 n}(K, \mathbb{Z})$ free of rank $k$ which admits a Sasakian metric of positive Ricci curvature. Furthermore, $K$ is diffeomorphic to one of the manifolds

$$
\# k\left(S^{2 n} \times S^{2 n+1}\right), \quad \#(k-1)\left(S^{2 n} \times S^{2 n+1}\right) \# T, \quad \# k\left(S^{2 n} \times S^{2 n+1}\right) \# \Sigma^{4 n+1},
$$

where $T=T_{1}\left(S^{2 n+1}\right)$ is the unit tangent bundle of $S^{2 n+1}$, and $\Sigma^{4 n+1}$ is the Kervaire sphere. For $k=1$ the manifolds

$$
S^{2 n} \times S^{2 n+1}, \quad\left(S^{2 n} \times S^{2 n+1}\right) \# \Sigma^{4 n+1}, \quad T
$$

all admit Sasakian metrics with positive Ricci curvature. If $n=1,3$ then $\# k\left(S^{2 n} \times\right.$ $S^{2 n+1}$ ) admits a Sasakian metric with positive Ricci curvature for all $k$.

The result here for $n=1$ was given previously in [13]. For highly connected rational homology spheres we have: 
Theorem 3 Every (2n-2)-connected oriented (4n-1)-manifold that is the boundary of a parallelizable manifold whose homology group $H_{2 n-1}(K, \mathbb{Z})$ is isomorphic to $\mathbb{Z}_{3}$ admits Sasakian metrics with positive Ricci curvature. There are precisely $2\left|b P_{4 n}\right|$ such smooth oriented manifolds.

There are two distinct oriented topological manifolds in this theorem and they are distinguished by their linking form in $H_{2 n-1}(K, \mathbb{Z}) \approx \mathbb{Z}_{3}$. However, they are equivalent as non-oriented manifolds. Moreover, each oriented manifold is comprised of $\left|b P_{4 n}\right|$ distinct oriented diffeomorphism types.

\section{Highly connected manifolds}

The most obvious subclass of highly connected manifolds are the homotopy spheres which were studied in detail in the seminal paper of Kervaire and Milnor [27]. We briefly summarize their results. Kervaire and Milnor defined an Abelian group $\Theta_{n}$ which consists of equivalence classes of homotopy spheres of dimension $n$ that are equivalent under oriented h-cobordism. By Smale's famous h-cobordism theorem [40] (see also Milnor [33]) this implies equivalence under oriented diffeomorphism. The group operation on $\Theta_{n}$ is connected sum. Now $\Theta_{n}$ has an important subgroup $b P_{n+1}$ which consists of equivalence classes of those oriented homotopy spheres which are the boundary of a parallelizable manifold. It is the subgroup $b P_{2 n}$ that is important for us in the present work. Kervaire and Milnor proved:

(i) $b P_{2 m+1}=0$.

(ii) $b P_{4 m}(m \geqslant 2)$ is cyclic of order $2^{2 m-2}\left(2^{2 m-1}-1\right)$ times the numerator of $\left(\frac{4 B_{m}}{m}\right)$, where $B_{m}$ is the $m$-th Bernoulli number. Thus, for example $\left|b P_{8}\right|=$ $28,\left|b P_{12}\right|=992,\left|b P_{16}\right|=8128,\left|b P_{20}\right|=130,816$.

(iii) $b P_{4 m+2}$ is either 0 or $\mathbb{Z}_{2}$.

Determining which $b P_{4 m+2}$ is $\{0\}$ and which is $\mathbb{Z}_{2}$ has proven to be difficult in general, and is still not completely understood. If $m \neq 2^{i}-1$ for any $i \geqslant 3$, then Browder [17] proved that $b P_{4 m+2}=\mathbb{Z}_{2}$. However, $b P_{4 m+2}$ is the identity for $m=1,3,7,15$ (Mahowald and Tangora [32] and Barratt, Jones and Mahowald [2]). See Lance [31] for a recent survey of results in this area and complete references. The answer is still unknown in the remaining cases. Using surgery Kervaire was the first to show that there is an exotic sphere in dimension 9 . His construction works in all dimensions of the form $4 m+1$, but as just discussed they are not always exotic.

In analogy with the Kervaire-Milnor group $b P_{2 n}$, Durfee [20] defined the group $B P_{2 n}$. Actually, he first defined this as a semigroup in [19], and later in [20] with the same notation denoted the corresponding Grothendieck group. Thus, we have: 
Definition 1.1 For $n \geqslant 3$ let $S B P_{2 n}$ denote the semigroup of oriented diffeomorphism classes of closed oriented $(n-2)$-connected $(2 n-1)-$ manifolds that bound parallelizable manifolds, and let $\mathrm{BP}_{2 n}$ denote its Grothendieck completion.

As with $b P_{2 n}$ mulitiplication in $S B P_{2 n}$ is the connected sum operation, and the standard sphere is a two-sided identity. Thus, $S B P_{2 n}$ is a monoid. Furthermore, the Kervaire and Milnor group $b P_{2 n}$ is a subgroup of $B P_{2 n}$ again by Smale's h-cobordism theorem. Unless otherwise stated we shall heretofore assume that $n \geqslant 3$, and that "manifold" will mean oriented manifold. We are mainly interested in those highly connected manifolds that can be realized as links of isolated hypersurface singularities defined by weighted homogeneous polynomials, so we have:

Definition 1.2 We denote by $W H P_{2 n-1}$ the set of oriented diffeomorphism classes of smooth closed oriented $(2 n-1)$ dimensional manifolds that can be realized as the link of an isolated hypersurface singularity of a weighted homogeneous polynomial in $\mathbb{C}^{n+1}$.

It is easy to see that elements of $W H P_{2 n-1}$ enjoy some nice properties.

Theorem 1.3 Let $M \in W H P_{2 n-1}$. Then

(i) $M$ is highly connected, that is, it is $(n-2)$-connected.

(ii) $M$ is the boundary of a compact $(n-1)$-connected parallelizable manifold $V$ of dimension $2 n$ with $H_{n}(V, \mathbb{Z})$ free.

(iii) If $n$ is even the $(n-1)$ st Betti number $b_{n-1}(M)$ is even.

(iv) $M$ is a spin manifold.

Proof Parts (i), (ii) and (iv) are well known. By [3] $M$ admits a Sasakian structure, so its odd Betti numbers are even up to the middle dimension by a well known result of Fujitani and Blair-Goldberg (cf [8]) which proves (iii).

From (i) and (ii) of Theorem 1.3 one sees that there is a map

$$
\Phi: W H P_{2 n-1} \longrightarrow B P_{2 n}
$$

which is the composition of the inclusion $W_{H P} P_{2 n-1} \hookrightarrow S B P_{2 n}$ with the natural semigroup homomorphism $S B P_{2 n} \longrightarrow B P_{2 n}$. The image $\Phi\left(W H P_{2 n-1}\right)$ is a subset of $B P_{2 n}$, and by (iii) of Theorem 1.3 it is a proper subset at least when $n$ is even. This can be contrasted with $b P_{2 n}$ which by a result of Brieskorn [16] satisfies $b P_{2 n} \cap$ 
$\Phi\left(W H P_{2 n-1}\right)=b P_{2 n}$ if $n \geqslant 3$. Notice, however, that $\Phi\left(W H P_{2 n-1}\right)$ is not generally a submonoid.

We now discuss invariants that distinguish elements of $B P_{2 n}$. First, by Poincaré duality the only non-vanishing homology groups occur in dimension $0, n-1, n$ and $2 n-1$. Moreover, $H_{n}(K, \mathbb{Z})$ is free and rank $H_{n}(K, \mathbb{Z})=\operatorname{rank} H_{n-1}(K, \mathbb{Z})$. Thus, our first invariant is the rank of $H_{n-1}(K, \mathbb{Z})$, so we define

$$
B P_{2 n}(k)=\left\{K \in B P_{2 n} \mid \operatorname{rank} H_{n-1}(K, \mathbb{Z})=k\right\} .
$$

This provides $B P_{2 n}$ with a grading, namely

$$
B P_{2 n}=\bigoplus_{k} B P_{2 n}(k)
$$

which is compatible with multiplication in $B P_{2 n}$ in the sense that

$$
\times: B P_{2 n}\left(k_{1}\right) \times B P_{2 n}\left(k_{2}\right) \longrightarrow B P_{2 n}\left(k_{1}+k_{2}\right) .
$$

Note that $B P_{2 n}(0)$ is the submonoid of highly connected rational homology spheres, and that $B P_{2 n}(k)$ is a $b P_{2 n}$-module.

The remaining known invariants (Wall [42], Durfee [19; 20]) are a linking form on the torsion subgroup of $H_{n-1}(K)$ and a quadratic invariant on the $2 n$-manifold whose boundary is $K$. The precise nature of these invariants depends on whether $n$ is even or odd. For the case $n$ even Durfee [20] shows that for $n \geqslant 3$ and $n \neq 4,8$ there is an exact sequence

$$
0 \longrightarrow b P_{2 n} \longrightarrow B P_{2 n} \stackrel{\Psi}{\longrightarrow} \mathbb{Z} \oplus K Q(\mathbb{Z}) \longrightarrow 0,
$$

where $K Q(\mathbb{Z})$ denotes the Grothendieck group of regular bilinear form modules over $\mathbb{Z}$. Let us describe the map $\Psi$. The projection onto the first factor is just the rank of $H_{n-1}(K)$ while the projection onto the second factor is Wall's quadratic form [42] which is essentially the classical linking form $b$ on the torsion subgroup of $H_{n-1}(K)$. Any two manifolds $K_{1}, K_{2} \in B P_{2 n}$ such that $\Psi\left(K_{1}\right)=\Psi\left(K_{2}\right)$ differ by a homotopy sphere, ie, there is $\Sigma \in b P_{2 n}$ such that $K_{2} \approx K_{1} \# \Sigma$ where $\approx$ means diffeomorphic. It is well known (Brieskorn [16]) that for $n$ even the elements $\Sigma \in b P_{2 n}$ are determined by the signature of $V$. This completes the diffeomorphism classification for $n \neq 4,8$ even. The cases $n=4,8$ are more complicated (Wilkens [43], Crowley [18]). Now, in addition to the group $H_{n-1}(K)$ and the linking form $b$, there is an obstruction cocycle $\hat{\beta} \in H^{n}\left(K, \pi_{n-1}(S O)\right) \approx H^{n}(K, \mathbb{Z})$. The tangent bundle of $K$ restricted to the $(n-1)$-skeleton is trivial and $\hat{\beta}$ gives the obstruction to triviality on the $n$-skeleton. If the torsion subgroup of $H_{n-1}(K)$ has odd order, then up to decomposability these are all the invariants. However, if the torsion subgroup of $H_{n-1}(K)$ has even order, 
things are even more complicated, and the analysis in [43] was not complete. It was recently completed in [18]. The important point for us is that if the torsion subgroup of $H_{n-1}(K)$ vanishes, $K$ is determined completely up to diffeomorphism by the rank of $H_{n-1}(K)$. Summarizing we have:

Theorem 1.4 Let $M$ be a highly connected manifold in $B P_{4 n}$ such that $H_{2 n-1}(M, \mathbb{Z})=\mathbb{Z}^{k}$. Then $M$ is diffeomorphic to $k \#\left(S^{2 n-1} \times S^{2 n}\right) \# \Sigma^{4 n-1}$ for some $\Sigma^{4 n-1} \in b P_{4 n}$.

Notice that by a well-known result of Fujitani and Blair-Goldberg (cf [8]) $k \#\left(S^{2 n-1} \times\right.$ $\left.S^{2 n}\right) \# \Sigma^{4 n-1}$ can admit a Sasakian structure only if $k$ is even.

For the case $n$ odd the diffeomorphism classification was obtained by Wall [42], but for our purposes, the presentation in [19] is more convenient. Let $K \in B P_{2 n}$ with $K=\partial V$, where $V$ can be taken as $(n-1)$-connected and parallelizable. In this case the key invariant is a $\mathbb{Z}_{2}$-quadratic form

$$
\psi: H_{n}(V, \mathbb{Z}) / 2 H_{n}(V, \mathbb{Z}) \longrightarrow \mathbb{Z}_{2}
$$

defined as follows: Let $X$ be an embedded $n$-sphere in $V$ that represents a non-trivial homology class in $H_{n}(V, \mathbb{Z})$, and let $[X]$ denote its image in $H_{n}(V, \mathbb{Z}) / 2 H_{n}(V, \mathbb{Z})$. Then $\psi([X])$ is the characteristic class in the kernel $\operatorname{ker}\left(\pi_{n-1}\left(S O(n) \longrightarrow \pi_{n-1}(S O)\right) \approx \mathbb{Z}_{2}\right.$ of the normal bundle of $X$. Let $\operatorname{rad} \psi$ be the radical of $\psi$, ie, the subspace of the $\mathbb{Z}_{2}$-vector space $H_{n}(V, \mathbb{Z}) / 2 H_{n}(V, \mathbb{Z})$ where $\psi$ is singular. Then Durfee [19] (see also [21]) proves:

Theorem 1.5 Let $K_{i} \in B P_{2 n}$ for $i=1,2$ with $n \geqslant 3$ odd be boundaries of parallelizable $(n-1)$-connected $2 n$ manifolds $V_{i}$ with $\mathbb{Z}_{2}$ quadratic forms $\psi_{i}$. Suppose that $H_{n-1}\left(K_{1}, \mathbb{Z}\right) \approx H_{n-1}\left(K_{2}, \mathbb{Z}\right)$, then

(i) if $n=3$ or 7 , then $K_{1}$ and $K_{2}$ are diffeomorphic;

(ii) if the torsion subgroups of $H_{n-1}\left(K_{i}, \mathbb{Z}\right)$ have odd order and $\psi_{i} \mid \operatorname{rad} \psi_{i} \equiv 0$ for $i=1,2$, then $K_{1} \approx K_{2} \#\left(c\left(\psi_{1}\right)+c\left(\psi_{2}\right)\right) \Sigma$, where $c$ is the Arf invariant and $\Sigma$ is the Kervaire sphere, ie, the generator of $b P_{2 n}$;

(iii) if the torsion subgroups of $H_{n-1}\left(K_{i}, \mathbb{Z}\right)$ have odd order and $\psi_{i} \mid \operatorname{rad} \psi_{i} \not \equiv 0$ for $i=1,2$, then $K_{1} \approx K_{2} \approx K_{2} \# \Sigma$.

It is convenient to define $W_{2 n-1}(k)$ to be the subset of $W H P_{2 n-1}$ such that $H_{n-1}$ has rank $k$. Then (iii) of Theorem 1.3 implies $W H P_{4 n-1}(2 k+1)=\varnothing$, whereas we shall see that $W H P_{4 n-1}(2 k) \neq \varnothing$ as well as $W H P_{4 n+1}(k) \neq \varnothing$ for all $k$. Recently, in the case $n=3$, Kollár [29;30] has discovered strong restrictions on the torsion 
subgroups of $H_{2}(K, \mathbb{Z})$ in order that $K$ admit a Sasakian structure which implies that $\Phi\left(\mathrm{WHP}_{6}(0)\right)$ is a proper subset of $\mathrm{BP}_{6}(0)$. One certainly expects these types of restrictions to persist in higher dimension as well.

\section{Branched covers and periodicity}

In this section we discuss some results of Durfee and Kauffman [21] concerning the periodicity of branched covers. Let $K \subset S^{2 n+1}$ be a simple fibered knot or link $(n \geqslant 1)$, by which we mean an $(n-2)$ connected $(2 n-1)$ embedded submanifold of $S^{2 n+1}$ for which the Milnor fibration theorem holds. If $F$ is the Milnor fiber of the fibration $\phi: S^{2 n+1}-K \longrightarrow S^{1}$ then the monodromy map $h: H_{n}(F) \longrightarrow H_{n}(F)$ is a fundamental invariant of the link $K$. Let $K_{k}$ be a $k$-fold cyclic branched cover of $S^{2 n+1}$ branched along $K$. Then Durfee and Kauffman [21] show that there is an exact sequence

$$
H_{n}(F) \stackrel{11+h+\cdots+h^{k-1}}{\longrightarrow} H_{n}(F) \longrightarrow H_{n}\left(K_{k}\right) \longrightarrow 0 .
$$

So homologically $K_{k}$ is determined by the cokernel of the map $11+h+\cdots+h^{k-1}$. Now suppose that $K$ is a rational homology sphere and that the monodromy map $h$ of $K$ has period $d$. Then since $1-h$ is invertible, $1+h+\cdots+h^{d-1}$ is the zero map in (2-1), and this determines the homology of $K_{d}$. Summarizing we have:

Lemma 2.1 (Durfee-Kauffman) Let $K$ be a fibered knot in $S^{2 n+1}$ which is a rational homology sphere such that the monodromy map has period $d$. Suppose further that $K_{k}$ is a $k$-fold cyclic cover of $S^{2 n+1}$ branched along $K$. Then

(i) $H_{n}\left(K_{d}\right) \approx H_{n}(F) \approx \mathbb{Z}^{\mu}$ where $\mu$ is the Milnor number of $K$.

(ii) $H_{*}\left(K_{k+d}\right) \approx H_{*}\left(K_{k}\right)$ for all $k>0$.

(iii) $H_{*}\left(K_{d-k}\right) \approx H_{*}\left(K_{k}\right)$ for all $0<k<d$.

Notice that (i) determines a large class of $n-1$ connected $2 n+1$-manifolds whose middle homology group $H_{n}$ is free, and in certain cases this determines the manifold up to homeomorphism. Items (ii) and (iii) give a homological periodicity.

Durfee and Kauffman also show that there are both homeomorphism and diffeomorphism periodicities in the case that $n$ is odd and $n \neq 1,3,7$. In particular in this case, when the link $K$ is a rational homology sphere whose monodromy map has period $d, K_{k+d}$ is homeomorphic to $K_{k}$. To obtain the diffeomorphism periodicity let $\sigma_{k}$ denote the signature of the intersection form on the Milnor fiber $F_{k}$. Again assuming that $K$ is a rational homology sphere and $h$ has periodicity $d$, one finds that $K_{k+d}$ is diffeomorphic to $\frac{\sigma_{d+1}}{8} \Sigma \# K_{k}$ where $\frac{\sigma_{d+1}}{8} \Sigma$ denotes $\frac{\sigma_{d+1}}{8}$ copies of the Milnor sphere $\Sigma$. Here we state the slightly more general theorem of Durfee [20, Theorem 6.4]: 
Theorem 2.2 For even $n \neq 2,4,8$ let $K_{i}$ be $(n-2)$-connected manifolds that bound parallelizable manifolds $V_{i}$, with $i=1$, 2. Suppose that the quadratic forms of $K_{i}$ are isomorphic and $H_{n-1}\left(K_{1}, \mathbb{Z}\right) \approx H_{n-1}\left(K_{2}, \mathbb{Z}\right)$. Then $\sigma\left(V_{2}\right)-\sigma\left(V_{1}\right)$ is divisible by 8 , and $K_{2}$ is diffeomorphic to $K_{1} \# \frac{1}{8}\left(\sigma\left(V_{2}\right)-\sigma\left(V_{1}\right)\right) \Sigma$ where $\sigma(V)$ is the Hirzebruch signature of $V$.

Remark 2.1 [20, Theorem 6.4] as well as [21, Theorem 5.3] exclude the cases $n=4$ and 8. However, it follows from [43] and [18] that the diffeomorphism classification still holds in these cases since the links we are considering here have no element of even order in the torsion subgroup of $H_{n-1}$ (In fact the torsion subgroup vanishes in the case above). This remark also pertains to the discussion for Theorem 3 below.

\section{Positive Ricci curvature on links}

Recall [13] that a Sasakian structure $(\xi, \eta, \Phi, g)$ is positive if the basic Chern class $c_{1}\left(\mathcal{F}_{\xi}\right)$ of the characteristic foliation $\mathcal{F}_{\xi}$ is positive. The importance of positive Sasakian structures comes from Theorem A of [13] which states that they give rise to Sasakian metrics with positive Ricci curvature. An important ingredient in the proof of this result is the 'transverse Yau theorem' of El Kacimi-Alaoui [22], or equivalently for the cases at hand, the orbifold version of Yau's theorem. Now there is a natural induced Sasakian structure on the link of a hypersurface singularity of a weighted homogeneous polynomial [3]. Combining this with 'orbifold adjunction theory' [8] we obtain:

Theorem 3.1 Let $L_{f}$ be the link of an isolated hypersurface singularity of a weighted homogeneous polynomial $f$ of degree $d$ and weight vector $\mathbf{w}$. Suppose further that $|\mathbf{w}|-d>0$. Then $L_{f}$ admits a Sasakian metric with positive Ricci curvature.

It is a simple task to construct positive Sasakian structures on links by increasing the dimension.

Proposition 3.2 Let $L_{f}$, be the link of a weighted homogeneous polynomial $f^{\prime}\left(z_{2}, \cdots, z_{n}\right)$ in $n-1$ variables with weight vector $\mathbf{w}^{\prime}$ and degree $d^{\prime}$. Assume that the origin in $\mathbb{C}^{n-1}$ is the only singularity so that $L_{f^{\prime}}$ is smooth. Consider the weighted homogeneous polynomial

$$
f=z_{0}^{2}+z_{1}^{2}+f^{\prime}
$$

of degree $d=\operatorname{lcm}\left(2, d^{\prime}\right)$. Then the link $L_{f}$ admits a Sasakian structure with positive Ricci curvature and $b_{n-1}\left(L_{f}\right)=b_{n-3}\left(L_{f^{\prime}}\right)$. 
Proof There are two cases. If $d^{\prime}$ is odd then the weight vector of $f$ is $\mathbf{w}=$ $\left(d^{\prime}, d^{\prime}, 2 \mathbf{w}^{\prime}\right)$, whereas, if $d^{\prime}$ is even, then $\mathbf{w}=\left(\frac{d^{\prime}}{2}, \frac{d^{\prime}}{2}, \mathbf{w}^{\prime}\right)$. In the first case we have $|\mathbf{w}|-d=d^{\prime}+d^{\prime}+2\left|\mathbf{w}^{\prime}\right|-2 d^{\prime}=2\left|\mathbf{w}^{\prime}\right|>0$, while in the second case $|\mathbf{w}|-d=\frac{d^{\prime}}{2}+\frac{d^{\prime}}{2}+\left|\mathbf{w}^{\prime}\right|-d^{\prime}=\left|\mathbf{w}^{\prime}\right|>0$. In either case $L_{f}$ admits a Sasakian metric with positive Ricci curvature by Theorem 3.1. The equality of Betti numbers is well known and follows from a theorem of Sebastiani and Thom [38; 26].

We note that it is easy to see that the appearance of the two 2's in $f$ implies that the klt conditions used to imply the existence of Sasakian-Einstein metrics [9; 7] cannot be satisfied. So we can say nothing at present about the existence of Sasakian-Einstein metrics on these links.

\section{Proofs of Theorems 1,2 and 3}

The links that we need to prove Theorems 1-3 involve Brieskorn-Pham polynomials of the form

$$
f_{p, q}=z_{0}^{p}+z_{1}^{q}+z_{2}^{2}+\cdots+z_{n}^{2} .
$$

The link associated with $f_{p, q}$ is

$$
L_{p, q}=\left\{f_{p, q}=0\right\} \cap S^{2 n+1} .
$$

By Proposition 3.2 all such links admit Sasakian metrics with positive Ricci curvature. One can view $L_{p, q}$ as a $p$-fold branched cover of $S^{2 n-1}$ branched over the link $L_{q}$ defined by the polynomial

$$
f_{q}=z_{1}^{q}+z_{2}^{2}+\cdots+z_{n}^{2}
$$

Proof of Theorem 1 Here we need the link $L_{2(2 k+1), 2 k+1}$, ie $p=2(2 k+1), q=$ $2 k+1$, with $n$ even (here $n$ corresponds to $2 n$ in the statement of the theorem). In this case the degree of $L_{2(2 k+1), 2 k+1}$ is $d=2(2 k+1)$ which is the period of the monodromy map of the link $L_{2 k+1}$. Furthermore, $L_{2 k+1}$ is a homotopy sphere by the Brieskorn Graph Theorem [16] or [8]. Now the link $L_{2(2 k+1), 2 k+1}$ is a $2(2 k+1)$ branched cover of $S^{2 n-1}$ branched over $L_{2 k+1}$, so by item (i) of Lemma 2.1, we have

$$
H_{n-1}\left(L_{2(2 k+1), 2 k+1}, \mathbb{Z}\right) \approx H_{n}\left(L_{2(2 k+1), 2 k+1}, \mathbb{Z}\right) \approx \mathbb{Z}^{\mu}=\mathbb{Z}^{2 k} .
$$


Here $\mu$ is the Milnor number [34] of the link $L_{2 k+1}$ which is easily computed by the formula for Brieskorn polynomials, namely

$$
\mu=\prod_{i=1}^{n}\left(a_{i}-1\right)=(2 k+1-1) \cdot 1 \cdots 1=2 k .
$$

Remark 4.1 Notice that the link $L_{2(2 k+1), 2 k+1}$ can be obtained by iterating Proposition 3.2 beginning with the Brieskorn manifold $M(2(2 k+1), 2 k+1,2)$ which is described in [35, Example 1 page 320]. As discussed there it is the total space of the circle bundle with Chern number -1 over a Riemann surface of genus $k$.

It now follows from Theorem 1.4 that $L_{2(2 k+1), 2 k+1}$ is diffeomorphic to $2 k \#\left(S^{n-1} \times\right.$ $\left.S^{n}\right) \# \Sigma^{4 n-1}$ for some $\Sigma^{4 n-1} \in b P_{4 n}$. (Here $n$ is as in the statement of the theorem.) We now use the periodicity results of Durfee and Kauffman to determine the diffeomorphism type. First we notice that Theorem 1.4 together with [21, Theorem 4.5] imply that for every positive integer $i$ and every positive integer $k$, the link $L_{2 i(2 k+1), 2 k+1}$ is homeomorphic to the connected sum $2 k \#\left(S^{n-1} \times S^{n}\right)$. The diffeomorphism types are determined by Theorem 2.2 ([20, Theorem 6.4], see also [21, Theorem 5.3]) together with Remark 2.1. Let $F_{i, k}$ denote the Milnor fibre of the link $L_{2 i(2 k+1), 2 k+1}$ and $\sigma\left(F_{i, k}\right)$ its Hirzebruch signature. Then Theorem 2.2 says that for each pair of positive integers $i, j$ there is a diffeomorphism

$$
L_{2 i(2 k+1), 2 k+1} \approx\left(\frac{\sigma\left(F_{i, k}\right)-\sigma\left(F_{j, k}\right)}{8} \Sigma\right) \# L_{2 j(2 k+1), 2 k+1},
$$

where $l \Sigma$ denotes the connected sum of $l$ copies of the Milnor sphere, and a minus sign corresponds to reversing orientation. Actually this formula follows from a signature periodicity result of Neumann as stated in [21, Theorem 5.2]. From Durfee's theorem the difference in signatures is always divisible by 8 , so this expression makes sense. Equation (4-3) can be iterated; so it is enough to consider the case $i=2$ and $j=1$. In order to determine how many distinct diffeomorphism types occur in (4-3), we need to compute the signature of the Milnor fibres. This is done in Appendix A. It is interesting to note that not all diffeomorphism types can be attained. This ends the proof of Theorem 1.

Proof of Theorem 2 Now we have $n$ odd (corresponding to $2 n+1$ in the statement of the theorem) and there are several cases. First we take $p=2(2 k+1), q=2 k+1$ as in the proof of Theorem 1. Again this leads to the link $L_{2(2 k+1), 2 k+1}$ with free homology satisfying Equation (4-2) except now $n$ is odd. Next we consider $q=2 k$ in Equation (4-1). The link $L_{2 k}$ of the Brieskorn-Pham polynomial $f_{2 k}=z_{1}^{2 k}+z_{2}^{2}+\cdots+z_{n}^{2}$ 
is a rational homology sphere by the Brieskorn Graph Theorem. Furthermore, its monodromy map has period $2 k$. Then choosing $p=2 k$ in Equation (4-1) the link $L_{2 k, 2 k}$ is $2 k$-fold branched cover over $S^{2 n+1}$ branched over the rational homology sphere $L_{2 k}$, so by item (i) of Lemma 2.1, we have

$$
H_{n-1}\left(L_{2 k, 2 k}, \mathbb{Z}\right) \approx H_{n}\left(L_{2 k, 2 k}, \mathbb{Z}\right) \approx \mathbb{Z}^{\mu}=\mathbb{Z}^{2 k-1} .
$$

These two cases now give links whose middle homology groups are free of arbitrary positive rank. However, unlike the case for $n$ even this does not determine the homeomorphism type unless $n=3,7$ in which case there is a unique diffeomorphism class. Indeed Theorem 1.5 implies we need to compute the quadratic form $\psi$, and this appears to be quite difficult in all but the simplest case. From Theorem 1.5 one can conclude [19] that if $M \in B P_{4 n+2}$ with $H_{2 n}(M, \mathbb{Z})$ free of rank one, then it is homeomorphic to $S^{2 n} \times S^{2 n+1}$ or the unit tangent bundle $T=T_{1}\left(S^{2 n+1}\right)$. (Now $n$ is as in the statement of the theorem). So the diffeomorphism types at most differ by an exotic Kervaire sphere $\Sigma^{4 n+1}$. Furthermore, $S^{2 n} \times S^{2 n+1}, T$ and $\left(S^{2 n} \times S^{2 n+1}\right) \# \Sigma^{4 n+1}$ generate the torsion-free submonoid of $B P_{4 n+2}$, there being relations in the monoid, namely, $T \# T=2 \#\left(S^{2 n} \times S^{2 n+1}\right)$ and $T \# \Sigma^{4 n+1}=T$ (Some further relations may exist depending on $n$ such as $\left.T_{1}\left(S^{3}\right) \approx S^{2} \times S^{3}\right)$. This proves the first statement in Theorem 2.

To prove the second statement we follow Durfee and Kauffman and consider a slightly different Brieskorn-Pham polynomial, namely $z_{0}^{2 k}+z_{1}^{2}+\cdots+z_{n}^{2}$. For $k=1$ we get as before a link $L_{2,2}$ whose middle homology group is free of rank one. Thus, it is diffeomorphic to one of the three generators above by (i) of Lemma 2.1. Now as $k$ varies we have a homological periodicity by (ii) and (iii) of Lemma 2.1. Durfee and Kauffman show that there is an 8-fold diffeomorphism periodicity, and they compute the $\psi$ invariant to show that

$$
\begin{gathered}
L_{2,2} \approx T, \quad L_{4,2} \approx\left(S^{2 n} \times S^{2 n+1}\right) \# \Sigma^{4 n+1}, \\
L_{6,2} \approx T \# \Sigma^{4 n+1} \approx T, \quad L_{8,2} \approx S^{2 n} \times S^{2 n+1} .
\end{gathered}
$$

This proves Theorem 2 .

Proof of Theorem 3 This is essentially a corollary of [20, Proposition 7.2] where Durfee considers the link $K_{k}$ of the Brieskorn-Pham polynomial $z_{0}^{k}+z_{1}^{3}+z_{2}^{2}+$ $\cdots+z_{n}^{2}$ for even $n \geqslant 4$. He shows that $H_{n}\left(K_{2}, \mathbb{Z}\right) \approx H_{n}\left(K_{4}, \mathbb{Z}\right) \approx \mathbb{Z}_{3}$, but that $K_{2}$ and $K_{4}$ have inequivalent linking forms. Furthermore, $K_{6 l+2}$ is diffeomorphic to $K_{2} \#(-1)^{\frac{n}{2}} l \Sigma^{4 n-1}$ and $K_{6 l+4}$ is diffeomorphic to $K_{4} \#(-1)^{\frac{n}{2}} l \Sigma^{4 n-1}$ where $\Sigma^{4 n-1}=$ $K_{5}$ is the Milnor generator. 


\section{Appendix A Computing the signature}

There are several known methods for computing the signature of the Milnor fibre $F$ of a Brieskorn manifold in the case when $n$ is odd. This was first accomplished for homotopy spheres by Brieskorn [16] and developed further by Hirzebruch and Zagier [24; 25]. Our discussion follows that in [24]. Let $\mathbf{a} \in\left(\mathbb{Z}^{+}\right)^{n+1}$ and write $\mathbf{a}=\left(a_{0}, \cdots, a_{n}\right)$. Consider the Brieskorn manifold $M_{\mathbf{a}}$ defined by the link

$$
\left\{z_{0}^{a_{0}}+\cdots+z_{n}^{a_{n}}=0\right\} \cap S^{2 n+1} .
$$

The Milnor fibre $F_{\mathbf{a}}$ can be represented by the Brieskorn manifold

$$
\left\{\mathbf{z} \in \mathbb{C}^{n+1} \mid z_{0}^{a_{0}}+\cdots+z_{n}^{a_{n}}=1\right\} .
$$

For $n$ even the Hirzebruch signature of $F_{\mathbf{a}}$ is given by the function

$$
\begin{array}{r}
t(\mathbf{a})=\#\left\{\mathbf{x} \in \mathbb{Z}^{n+1} \mid 0<x_{k}<a_{k} \text { and } 0<\sum_{j=0}^{n} \frac{x_{k}}{a_{k}}<1 \quad \bmod 2\right\} \\
(\mathrm{A}-1) \quad-\#\left\{\mathbf{x} \in \mathbb{Z}^{n+1} \mid 0<x_{k}<a_{k} \text { and } 1<\sum_{j=0}^{n} \frac{x_{k}}{a_{k}}<2 \bmod 2\right\} .
\end{array}
$$

Using methods of Fourier analysis, Zagier has obtain the following formula for $t(\mathbf{a})$ :

$$
t(\mathbf{a})=\frac{(-1)^{\frac{n}{2}}}{N} \sum_{j=0}^{N-1} \cot \frac{\pi(2 j+1)}{2 N} \cot \frac{\pi(2 j+1)}{2 a_{0}} \cdots \cot \frac{\pi(2 j+1)}{2 a_{n}},
$$

where $N$ is any common multiple of the $a_{i}$ 's.

We now adapt this formula to treat the link of the Brieskorn-Pham polynomial of Equation (4-1) with $N=2(2 k+1)$, namely, $\mathbf{a}=(2(2 k+1), 2 k+1), 2 \cdots, 2)$. Notice that we can always take the $N$ in Zagier's formula (A-2) to be the same as the $N$ in Equation (4-1). In this case we shall denote $t$ (a) by $t_{d}$ since the degree $d=2(2 k+1)$ is the periodicity as well. Likewise, we denote by $t_{2 d}$ the signature $t$ (a) with $\mathbf{a}=(4(2 k+1), 2 k+1), 2 \cdots, 2)$. We find

$$
t_{d}=\frac{(-1)^{\frac{n}{2}}}{4 k+2} \sum_{j=0}^{4 k+1}(-1)^{j} \cot ^{2} \frac{\pi(2 j+1)}{8 k+4} \cot \frac{\pi(2 j+1)}{4 k+2},
$$

and

$$
t_{2 d}=\frac{(-1)^{\frac{n}{2}}}{8 k+4} \sum_{j=0}^{8 k+3}(-1)^{j} \cot ^{2} \frac{\pi(2 j+1)}{16 k+8} \cot \frac{\pi(2 j+1)}{4 k+2} .
$$




\begin{tabular}{|c|c|c|c|}
\hline \multicolumn{5}{|c|}{ Table 1 $2 k \#\left(S^{3} \times S^{4}\right)$} \\
\hline$k$ & $\tau_{k}$ & $D_{2}(k)$ & $\frac{D_{2}(k)}{\left|b P_{8}\right|}$ \\
\hline 1 & 1 & 28 & 1 \\
\hline 2 & 3 & 28 & 1 \\
\hline 3 & 10 & 14 & $\frac{1}{2}$ \\
\hline 4 & 15 & 14 & $\frac{1}{2}$ \\
\hline 5 & 21 & 28 & 1 \\
\hline 6 & 28 & 4 & $\frac{1}{7}$ \\
\hline 7 & 36 & 1 & $\frac{1}{28}$ \\
\hline 8 & 45 & 7 & $\frac{1}{4}$ \\
\hline 9 & 55 & 28 & 1 \\
\hline 10 & 210 & 28 & 1 \\
\hline 20 & 1176 & 2 & $\frac{1}{14}$ \\
\hline 48 & 1275 & 1 & $\frac{1}{28}$ \\
\hline 50 & 5050 & 28 & $\frac{1}{28}$ \\
\hline 100 & 123256 & 14 & $\frac{1}{2}$ \\
\hline 496 & 125250 & 14 & 1 \\
\hline 500 & & & 14 \\
\hline
\end{tabular}

We want to compute $\tau_{k}=\frac{\left|t_{2 d}-t_{d}\right|}{8}$. After some algebra we find that $(64 k+32) \tau_{k}$ equals

(A-3) $\sum_{j=0}^{8 k+3}(-1)^{j} \cot \frac{\pi(2 j+1)}{16 k+8}\left(\cot \frac{\pi(2 j+1)}{16 k+8}-\cot \frac{\pi(2 j+1)}{8 k+4}\right) \cot \frac{\pi(2 j+1)}{4 k+2}$.

Now $\tau_{k}$ is always an integer, and by (A-3) it is independent of $n$. We now define

$$
D_{n}(k)=\frac{\left|b P_{4 n}\right|}{\operatorname{gcd}\left(\tau_{k},\left|b P_{4 n}\right|\right)} .
$$

By Equation (4-3), $D_{n}(k)$ represents the number of distinct diffeomorphism types that can be represented by our construction. Using MAPLE we give two tables consisting of a list of $\tau_{k}$ and $D_{n}(k)$ together with the ratio

$$
\frac{D_{2}(k)}{\left|b P_{8}\right|}=\frac{1}{\operatorname{gcd}\left(\tau_{k},\left|b P_{4 n}\right|\right)}
$$




\begin{tabular}{|c|c|c|c|}
\hline \multicolumn{4}{|c|}{ Table $2 \quad 2 k \#\left(S^{5} \times S^{6}\right)$} \\
\hline$k$ & $\tau_{k}$ & $D_{3}(k)$ & $\frac{D_{3}(k)}{\left|b P_{12}\right|}$ \\
\hline 1 & 1 & 992 & 1 \\
\hline 2 & 3 & 992 & 1 \\
\hline 3 & 6 & 496 & $\frac{1}{2}$ \\
\hline 4 & 10 & 496 & $\frac{1}{2}$ \\
\hline 5 & 15 & 992 & 1 \\
\hline 6 & 21 & 992 & 1 \\
\hline 7 & 28 & 248 & $\frac{1}{4}$ \\
\hline 8 & 36 & 248 & $\frac{1}{4}$ \\
\hline 9 & 45 & 992 & 1 \\
\hline 10 & 55 & 992 & 1 \\
\hline 31 & 496 & 2 & $\frac{1}{496}$ \\
\hline 48 & 1176 & 124 & $\frac{1}{8}$ \\
\hline 50 & 1275 & 992 & 1 \\
\hline 62 & 1953 & 32 & $\frac{1}{31}$ \\
\hline 124 & 7750 & 16 & $\frac{1}{62}$ \\
\hline 248 & 30876 & 8 & $\frac{1}{124}$ \\
\hline 496 & 123256 & 4 & $\frac{1}{248}$ \\
\hline 500 & 125250 & 496 & $\frac{1}{2}$ \\
\hline 992 & 492528 & 2 & $\frac{1}{496}$ \\
\hline
\end{tabular}

for both the 7-manifolds \#2k $\left(S^{3} \times S^{4}\right)$ and the 11 -manifolds $\# 2 k\left(S^{5} \times S^{6}\right)$ for various values of $k$.

Notice that the prime factorization of $\left|b P_{4 n}\right|$ consists of high powers of two together with odd primes coming from the Bernoulli numbers. Since $\tau_{k}$ is independent of $n$, this gives rise to a bit of a pattern for the ratios $\frac{D_{n}(k)}{\left|b P_{4 n}\right|}$. It is obvious that for $k=1$ all possible diffeomorphism types occur, but this seems also to hold for $k=2$. It is of course true whenever $\left|b P_{4 n}\right|$ is relatively prime to 3 . If we look at the next case namely, $b P_{16}$, we see that $\left|b P_{16}\right|=8128=2^{6} \cdot 127$. Comparing this with $\left|b P_{12}\right|=992=2^{5} \cdot 31$, we see that the same ratios will occur for the case $\# 2 k\left(S^{7} \times S^{8}\right)$ as for $\# 2 k\left(S^{5} \times S^{6}\right)$ for $k=1, \cdots, 30$. It is interesting to contemplate whether the above gaps in the diffeomorphism types occur as a consequence of our method or whether they indicate 
an honest obstruction to the existence of positive Sasakian structures. At this stage we have no way of knowing.

Acknowledgements We thank Stephan Stolz for helpful conversations, and David Wraith for finding some typos and pointing out the need for some clarifications. We also owe thanks to Walter Neumann and the Editorial Board of G\&T as well as the anonomous referee for further clarifications and corrections. The second author would like to thank Max-Planck-Institut für Mathematik in Bonn for hospitality. During the preparation of this work the authors were partially supported by NSF grants DMS0203219 and DMS-0504367.

\section{References}

[1] D Barden, Simply connected five-manifolds, Ann. of Math. (2) 82 (1965) 365-385 MR0184241

[2] M G Barratt, J D S Jones, ME Mahowald, Relations amongst Toda brackets and the Kervaire invariant in dimension 62, J. London Math. Soc. (2) 30 (1984) 533-550 MR810962

[3] C P Boyer, K Galicki, New Einstein metrics in dimension five, J. Differential Geom. 57 (2001) 443-463 MR1882664

[4] CP Boyer, K Galicki, Rational homology 5-spheres with positive Ricci curvature, Math. Res. Lett. 9 (2002) 521-528 MR1928872

[5] CP Boyer, K Galicki, New Einstein metrics on $8 \#\left(S^{2} \times S^{3}\right)$, Differential Geom. Appl. 19 (2003) 245-251 MR2002662

[6] CP Boyer, K Galicki, Sasakian geometry, hypersurface singularities, and Einstein metrics, Rend. Circ. Mat. Palermo (2) Suppl. (2005) 57-87 MR2152356

[7] C P Boyer, K Galicki, Einstein metrics on rational homology spheres, J. Differential Geom. 74 (2006) 353-362

[8] C P Boyer, K Galicki, Sasakian geometry, Oxford Mathematical Monographs, Oxford University Press, to appear (2007)

[9] C P Boyer, K Galicki, J Kollár, Einstein metrics on spheres, Ann. of Math. (2) 162 (2005) 557-580 MR2178969

[10] CP Boyer, K Galicki, J Kollár, E Thomas, Einstein metrics on exotic spheres in dimensions 7, 11, and 15, Experiment. Math. 14 (2005) 59-64 MR2146519

[11] C P Boyer, K Galicki, M Nakamaye, Einstein metrics on rational homology 7-spheres, Ann. Inst. Fourier (Grenoble) 52 (2002) 1569-1584 MR1935557

[12] C P Boyer, K Galicki, M Nakamaye, Sasakian-Einstein structures on $9 \#\left(S^{2} \times S^{3}\right)$, Trans. Amer. Math. Soc. 354 (2002) 2983-2996 MR1897386 
[13] C P Boyer, K Galicki, M Nakamaye, On positive Sasakian geometry, Geom. Dedicata 101 (2003) 93-102 MR2017897

[14] CP Boyer, K Galicki, M Nakamaye, On the geometry of Sasakian-Einstein 5manifolds, Math. Ann. 325 (2003) 485-524 MR1968604

[15] C P Boyer, K Galicki, M Nakamaye, Sasakian geometry, homotopy spheres and positive Ricci curvature, Topology 42 (2003) 981-1002 MR1978045

[16] E Brieskorn, Beispiele zur Differentialtopologie von Singularitäten, Invent. Math. 2 (1966) 1-14 MR0206972

[17] W Browder, The Kervaire invariant of framed manifolds and its generalization, Ann. of Math. (2) 90 (1969) 157-186 MR0251736

[18] D Crowley, The classification of highly connected manifolds in dimension 7 and 15, $\mathrm{PhD}$ thesis, Indiana University (2001)

[19] A H Durfee, Diffeomorphism Classification of Isolated Hypersurfaces Singularities, $\mathrm{PhD}$ thesis, Cornell University (1971)

[20] A H Durfee, Bilinear and quadratic forms on torsion modules, Advances in Math. 25 (1977) 133-164 MR0480333

[21] A H Durfee, L Kauffman, Periodicity of branched cyclic covers, Math. Ann. 218 (1975) 157-174 MR0385872

[22] A El Kacimi-Alaoui, Opérateurs transversalement elliptiques sur un feuilletage riemannien et applications, Compositio Math. 73 (1990) 57-106 MR1042454

[23] K Grove, W Ziller, Cohomogeneity one manifolds with positive Ricci curvature, Invent. Math. 149 (2002) 619-646 MR1923478

[24] F Hirzebruch, Singularities and exotic spheres, from: "Séminaire Bourbaki, Vol. 10", Soc. Math. France, Paris (1995) Exp. No. 314, 13-32 MR1610436

[25] F Hirzebruch, D Zagier, The Atiyah-Singer theorem and elementary number theory, Mathematics Lecture Series 3, Publish or Perish, Boston (1974) MR0650832

[26] L H Kauffman, W D Neumann, Products of knots, branched fibrations and sums of singularities, Topology 16 (1977) 369-393 MR0488073

[27] M A Kervaire, J W Milnor, Groups of homotopy spheres. I, Ann. of Math. (2) 77 (1963) 504-537 MR0148075

[28] J Kollár, Einstein metrics on connected sums of $S^{2} \times S^{3}$, J. Differential Geom. to appear arXiv:math.DG/0402141

[29] J Kollár, Einstein metrics on five-dimensional Seifert bundles, J. Geom. Anal. 15 (2005) 445-476 MR2190241

[30] J Kollár, Circle actions on simply connected 5-manifolds, Topology 45 (2006) 643-671 MR2218760 
[31] T Lance, Differentiable structures on manifolds, from: "Surveys on surgery theory, Vol. 1", Ann. of Math. Stud. 145, Princeton Univ. Press (2000) 73-104 MR1747531

[32] M Mahowald, M Tangora, Some differentials in the Adams spectral sequence, Topology 6 (1967) 349-369 MR0214072

[33] J Milnor, Lectures on the h-cobordism theorem, Notes by L. Siebenmann and J. Sondow, Princeton University Press (1965) MR0190942

[34] J Milnor, Singular points of complex hypersurfaces, Annals of Mathematics Studies 61, Princeton University Press (1968) MR0239612

[35] J Milnor, On the 3-dimensional Brieskorn manifolds $M(p, q, r)$, from: "Knots, groups, and 3-manifolds (Papers dedicated to the memory of R H Fox)", (L P Neuwirth, editor), Annals of Mathematics Studies 84, Princeton University Press (1975) 175-225 MR0368009

[36] J Rosenberg, S Stolz, Metrics of positive scalar curvature and connections with surgery, from: "Surveys on surgery theory, Vol. 2", Annals of Mathematics Studies 149, Princeton Univ. Press (2001) 353-386 MR1818778

[37] L J Schwachhöfer, W Tuschmann, Metrics of positive Ricci curvature on quotient spaces, Math. Ann. 330 (2004) 59-91 MR2091679

[38] M Sebastiani, R Thom, Un résultat sur la monodromie, Invent. Math. 13 (1971) 90-96 MR0293122

[39] J-P Sha, D Yang, Positive Ricci curvature on the connected sums of $S^{n} \times S^{m}$, J. Differential Geom. 33 (1991) 127-137 MR1085137

[40] S Smale, On the structure of manifolds, Amer. J. Math. 84 (1962) 387-399 MR0153022

[41] C T C Wall, Classification of ( $n-1)$-connected $2 n$-manifolds, Ann. of Math. (2) 75 (1962) 163-189 MR0145540

[42] C T C Wall, Classification problems in differential topology. VI. Classification of ( $s-1)$ connected $(2 s+1)$-manifolds, Topology 6 (1967) 273-296 MR0216510

[43] D L Wilkens, Closed $(s-1)$-connected $(2 s+1)$-manifolds, $s=3$, 7, Bull. London Math. Soc. 4 (1972) 27-31 MR0307258

Department of Mathematics and Statistics, University of New Mexico

Albuquerque, NM 87131, USA

cboyer@math.unm.edu, galicki@math.unm.edu

Proposed: Gang Tian

Received: 5 September 2005

Seconded: Leonid Polterovich, Tobias Colding

Revised: 22 October 2006 